\title{
Hamburger Automatic Wrapping Machine
}

\author{
Reo Takeishia ${ }^{a}$ Yuhki Kitazono ${ }^{a, *}$

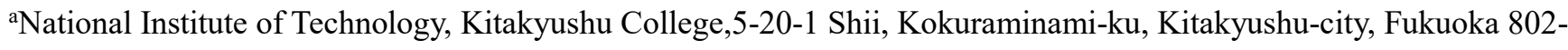 \\ 0985, Japan \\ *Corresponding Author: kitazono@kct.ac.jp
}

\begin{abstract}
Use 5 Servo motors to wrap the finished hamburger with paper. Set the paper and burger at the specified location and start operation. Create and control shapes using Arduino and universal plates. First, move each motor to the initial position (initial angle). Assign numbers to each motor and move them in order.
\end{abstract}

Keywords: Hamburger, Wrapping Machine, Servo Motor.

\section{Introduction}

Speaking of hamburgers, what company do you consider? We think McDonald's is the most representative. There, now, we decided to conduct research by listing McDonald's used by many people around the world. 1948, The McDonald brothers started in California, USA. Now it is the world's leading fast food restaurant. The reason why McDonald's are loved by many people is think the reasons for the popularity are the goodness of cost performance, the speed from ordering to product delivery, and the teste. Recently, we think the reasons for the popularity are the goodness of cost performance, the speed from ordering to product delivery, and the taste. Recently, mobile orders have been put in place, allowing customers to select and pay for items with their mobile phone, and stores receive the products. At that time, we thought about improving the efficiency of rotation in the store, so we decided to make a machine that automatically packs hamburgers.

Hamburgers are carefully packaged by the crew at each store. If this time could be shortened a little, the turnover in the shop would be higher than it is now.

Some people think that the turnover cannot be increased so much in a few seconds. Certainly, even if a person speeds up when rattling, the turnover rate will not change and the profit will not change. But even so, we think it would be better if the delivery time to the customer was reduced and the crew was a little easier.

And if the store is crowded, what if you could take this opportunity and allow the crew to do other things for the hamburger packaging time?

Each hamburger can save 3 seconds. Let's say that there are 100 times during the three-second, most popular time period. Then, about 5 minutes can be effectively used as a whole. The five minutes can be as long as 30 hours a year. If you think so, it means that you have increased the turnover rate for 30 hours in one year.

In addition, as at once it is 10 pieces of packaging that to the well that ten times of 300 hours it means that by increasing the rotation rate. Considering the whole country, the number of McDonald's in Japan is about 2,900, which means 870,000 hours. Then, when converting the time to self-sufficiency, the minimum wage ${ }^{(1)}$ in Fukuoka is 870,000 $\mathrm{x}$ about $841=73167,000$, and it is a calculation that profits at least about 73 million yen

This difference is likely to be very large on the whole but small on the whole.

Therefore, we propose hamburger automatic wrapping machine.

\section{Device Configuration}

Fig. 1 shows an operation image of the device. This device consists of a control unit based on Android UNO and a drive unit based on a servomotor. Users of the device start by placing a piece of wrapping paper on the device, placing a hamburger in the middle. When started, the hamburger is wrapped in paper by the attached servomotor. Finally, the device user removes the wrapped hamburger from the device.

The device waits for the user to place the next piece of paper and hamburger and press the start button. As mentioned above, the user places the paper and burger on the 


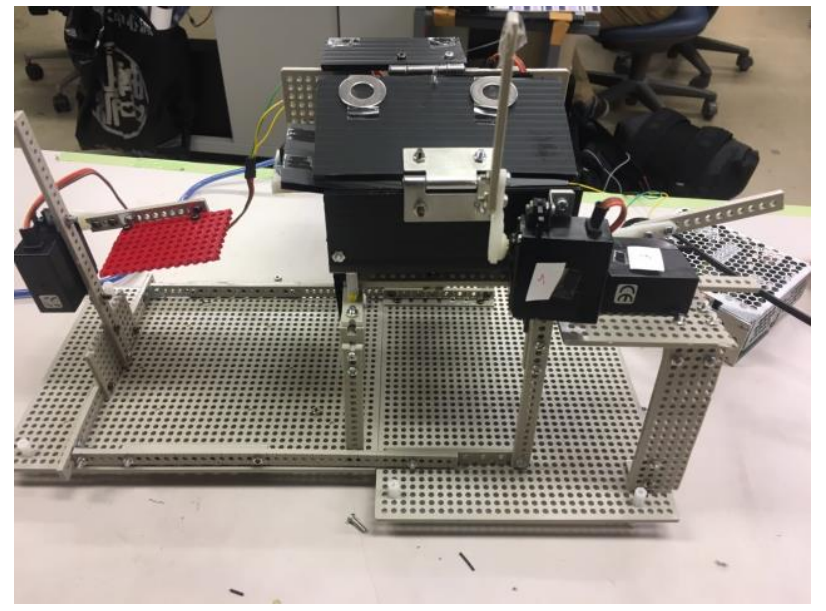

Fig. 1. Overview.

device and presses the start button. The device automatically wraps the burger and the user removes the wrapped burger from the device. By repeating this series of operations, the "wrapping" process of the hamburger is automated.

\subsection{Overview}

First, we made a device to wrap a burger. We drew how to wrap it on paper. The operation of wrapping the burger is to place the burger and wrapping paper in the receiving box and fold the paper using a plate from four directions. What we devised at this time was the shape of the plate. The size of each plate was changed to prevent the plates from overlapping when the right and left plates were operated simultaneously. Another problem when wrapping a burger is that simply folding the plate open will cause the paper to float. In order to prevent it, we made some arrangements for folding the plates from four directions. That is, when the paper is folded with the right hand, the paper is folded in the opposite direction with the left hand while supporting the paper with the right hand. The last operation was to turn it upside down. Then, the work of attaching the plate for receiving the burger that has come around so that the Nakano burger does not fall off the plate due to the vibration when it is turned over, and wrapping the burger off the plate is the end of the work of wrapping.

Next, we collected the materials to run the model created on paper. The first thing I needed was a motor. Three motors are used to fold paper. One motor to turn upside down. One motor to catch and drop the overturned burger. A total of five were used.

\subsection{Operating Procedure}

(1) Place the hamburger in the designated place.

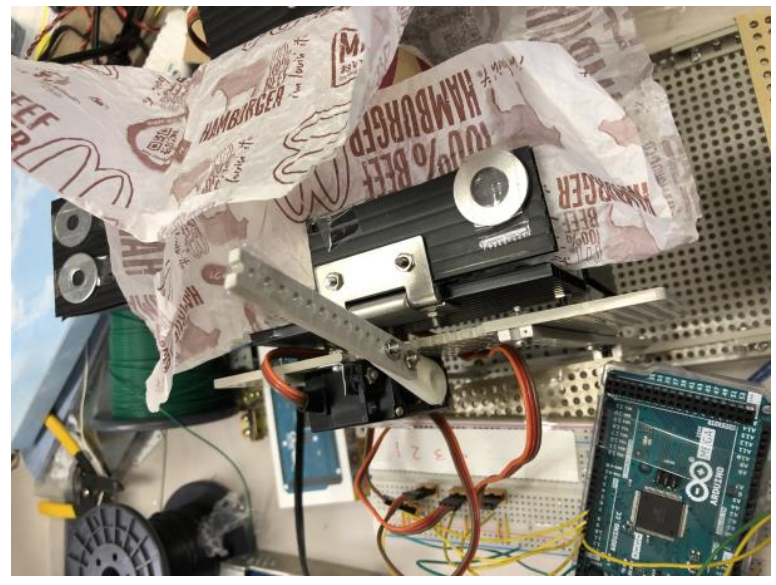

Fig. 2. Operation 1.

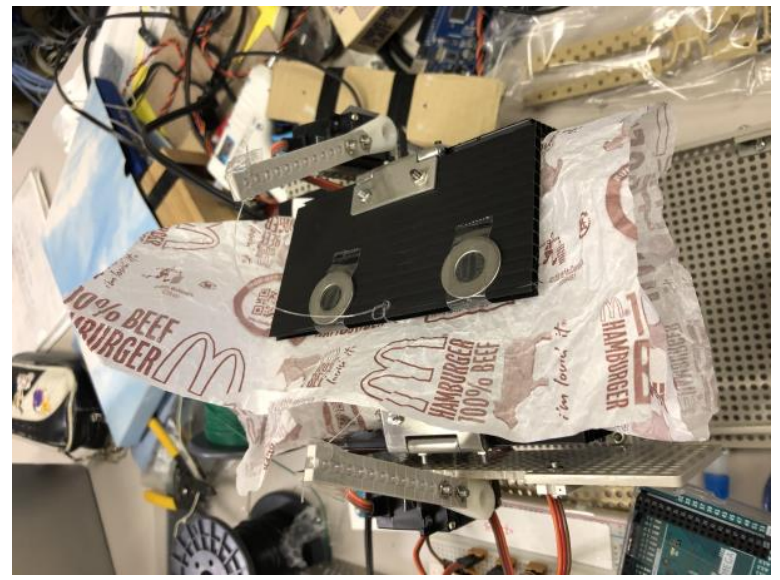

Fig. 3. Operation 2.

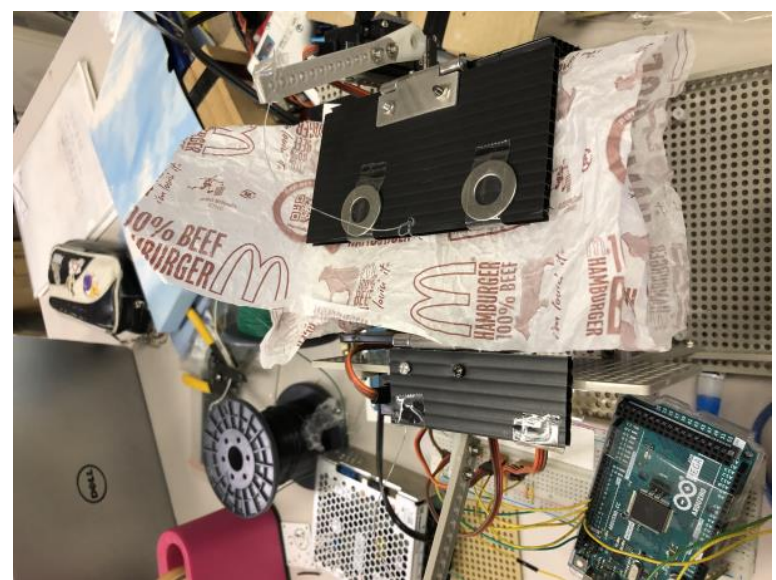

Fig. 4. Operation 3.

(2) Wrap the hamburger so that the paper is folded.

(3) Turn the hamburger upside down 180 degrees.

(4) Lower the plate (Servo5) that has received the hamburger.

The operation is shown in Figs. 2-10. 


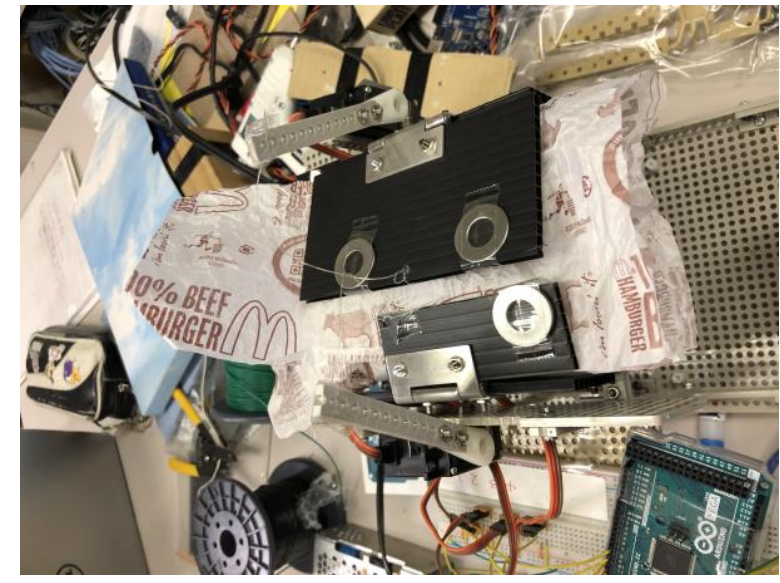

Fig. 5. Operation 4.

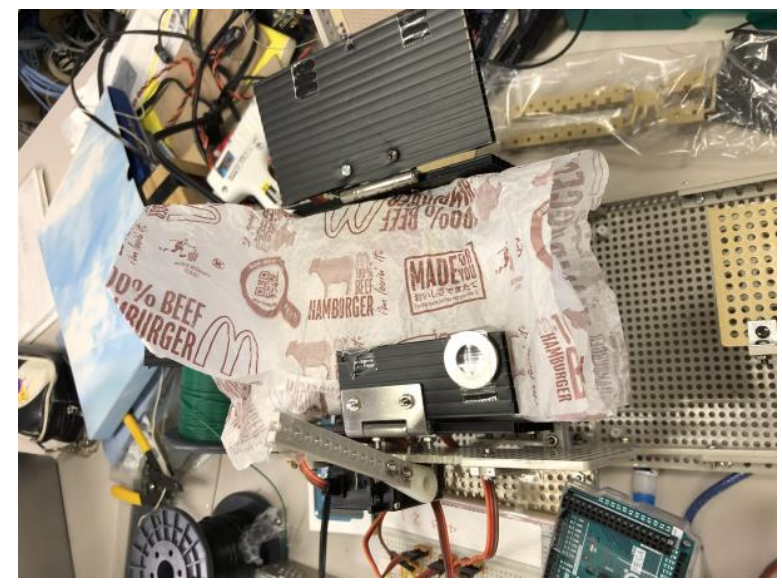

Fig. 6. Operation 5.

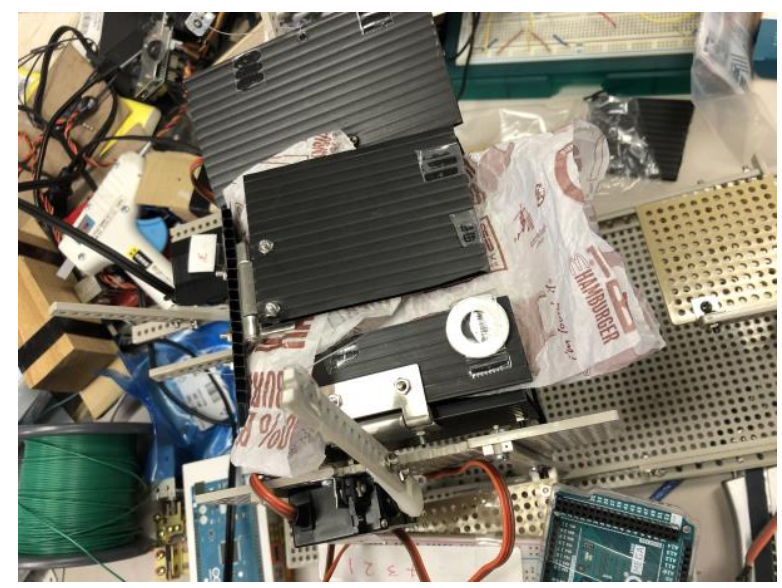

Fig. 7. Operation 6.

\section{Experiment}

In this experiment, the hamburger was wrapped automatically. After finishing the wrapping, the upper side of the hamburger bun could be turned up by rotating it 180

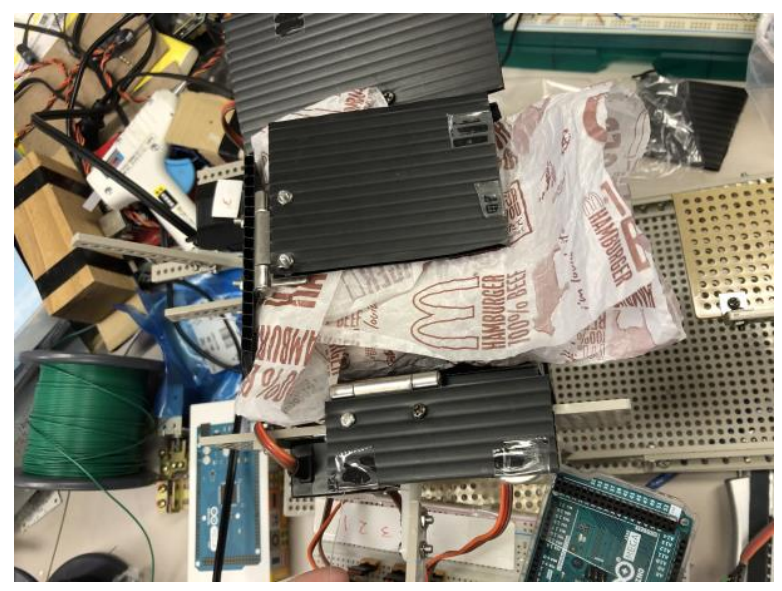

Fig. 8. Operation 7.

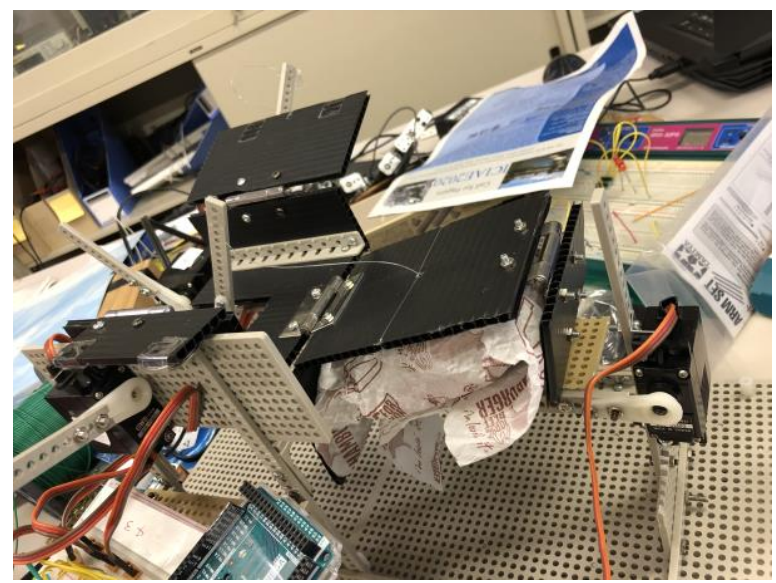

Fig. 9. Operation 8 .

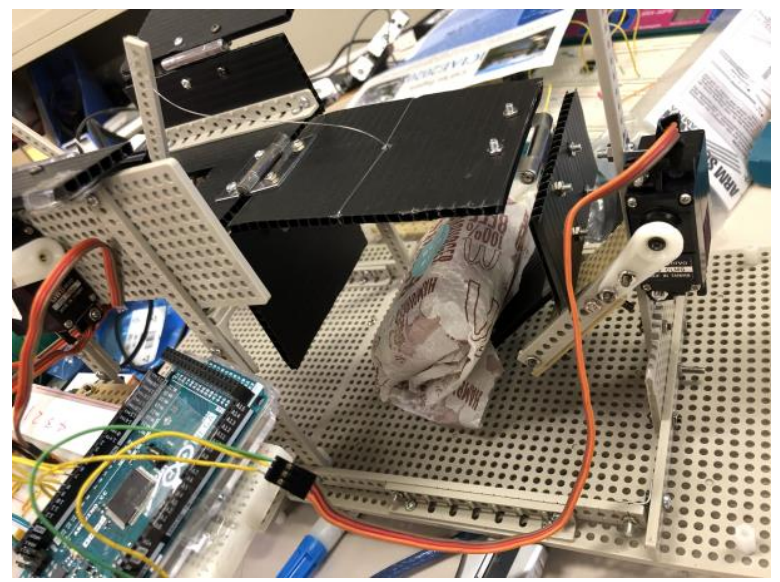

Fig. 10. Operation 9.

degrees.

However, the speed improvement, which was the purpose of this project, could not be achieved. It took about 40 seconds to wrap one hamburger. Also, the paper wrapped around the hamburger was loose, and the hamburger often came out of the paper when flipped 180 degrees. In addition, 
the hamburger that I dropped in the last move was difficult.

We would like to work on the following as future tasks.

(a) Reduction of waiting time between operations

(b) Apply pressure by controlling plate power when wrapping hamburger with paper

Raise the foot of the base and attach something like a slide to make the hamburger flow and make it easier to take.

\section{Conclusions}

The hamburger was automatically wrapped. It can be easily created by controlling with Arduino. The one created this time is still less complete, and there are many improvements and improvements, but the degree of completion can be improved accordingly. By continuing this improvement, we will continue to improve it with the goal of putting it into actual production lines ${ }^{(2)}$.

\section{References}

(1) Nationwide list of low wages by region

https://www.mhlw.go.jp/stf/seisakunitsuite/bunya/koyo u_roudou/roudoukijun/minimumichiran/

(2) Introduction of robots into the restaurant industry, which progresses from fast food https://www.technologyreview.jp/s/31917/roboticchefs-are-getting-better-if-you-like-fast-food/ 